\title{
Influence of the Reynolds Number on Transonic Tip Flow
}

\author{
Wuheng Feng, Yuxin Zhao, Qiancheng Wang, and Chenglong Wang $\mathbb{D}$ \\ College of Aerospace Science and Engineering, National University of Defense Technology, Changsha, Hunan 410073, China
}

Correspondence should be addressed to Chenglong Wang; wangchenglong@nudt.edu.cn

Received 4 June 2020; Revised 13 August 2020; Accepted 20 October 2020; Published 7 November 2020

Academic Editor: Jing-Lei Xu

Copyright (C) 2020 Wuheng Feng et al. This is an open access article distributed under the Creative Commons Attribution License, which permits unrestricted use, distribution, and reproduction in any medium, provided the original work is properly cited.

\begin{abstract}
The tip flows in modern gas turbines are primarily transonic under realistic conditions and significantly impact the overall thrust performance and safety of the turbines. This study is aimed at providing a deeper understanding of the mechanisms underlying and controlling the tip flow characteristics. Particle image velocimetry (PIV) and Schlieren and oil flow visualizations were performed to reveal the basic structure of the tip flow fields. A computational fluid dynamics model was developed, and the experimental results validated its accuracy. FLUENT 18.0 was employed to apply the Spalart-Allmaras turbulence model and perform two-dimensional calculations that furthered the investigation. The PIV and Schlieren visualization results indicated that the tip flow accelerated rapidly to the transonic level at the gap inlet separation when the gap pressure ratio exceeded 2.0. Furthermore, an oblique shock wave was generated when the transonic tip flow reattached and then reflected within the gap. The oil flow visualization provided the corresponding boundary layer behavior on the bottom wall. Additionally, the computation of the transonic tip flow with respect to various sizes and pressure values demonstrated that the Reynolds number is the key parameter that controls the gap flow field. The flow similarity existed as long as the Reynolds number remained constant. An in-depth analysis of the simulation improved the model performance at predicting the inlet separation size, discharge coefficient, and friction coefficient based on the Reynolds number. The study results provide a reference for the design and testing of engine blade gaps in realworld conditions.
\end{abstract}

\section{Introduction}

Gas turbines are thermal power equipment having strategic significance in industrial and economic activities and have been widely applied in a range of fields, including land power generation and aircraft and ship propulsion. During gas turbine operation, the gaps between the tips of the rotating turbine blades and stationary casing ensure high-speed blade rotation and prevent friction, as shown in Figure 1. The height of the tip gap is usually less than $2 \mathrm{~mm}$. Due to the pressure difference between the blades, hot gas leaks from the pressure side to the suction side over the blade tip. This tip flow significantly affects the thrust performance and stable operation of the engines. It is estimated that aerodynamic loss due to tip flow accounts for approximately $30 \%$ of the total stage loss [1]. The tip flow can also lead to an increase in the local heat flux in the high-temperature turbine blade or even damage the engine structures.
In traditional engines, the pressure difference across the tip is relatively small (i.e., the pressure ratio is $1.0-1.5$ ), and the flow in the gap is subsonic. As the turbine load is gradually increased to obtain greater turbine power and thermal efficiency, a larger pressure difference occurs across the tip (i.e., the pressure ratio increases to 1.5-3.0). Hence, the flow across the gap becomes transonic. Chen et al. [3] conducted experimental and numerical studies using a simplified tip gap model and suggested that the peak Mach number in the gap is 1.4 when the exit Mach number is 1.0. Numerical and experimental studies conducted by Wheeler et al. [4] on a high-pressure turbine blade showed that the flows in the gap were mainly transonic under realworld engine conditions.

The characteristics of the subsonic and the transonic gap flows differ significantly, and few studies have investigated the nature of transonic gap flow, especially experimentally. Specifically, the computational fluid dynamics (CFD) 


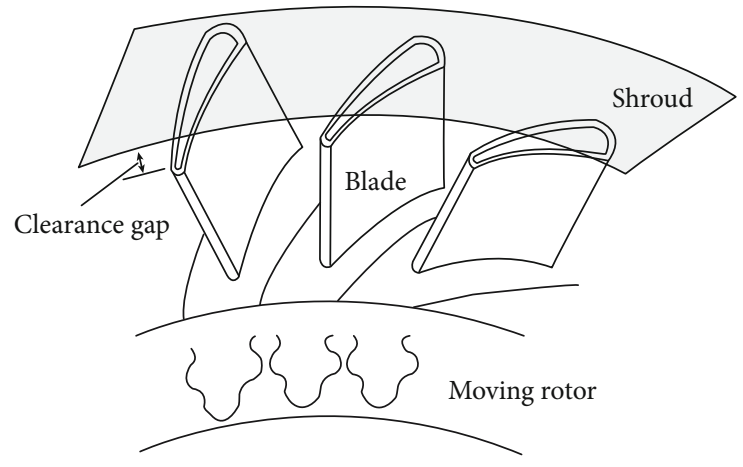

Figure 1: Schematic of a tip gap [2].

predictions of Coull et al. [5] showed that transonic flow occurs in the rear portion of the tip and is largely determined by the tip gap configuration. The numerical analysis of Saha et al. [6] demonstrated that the pressure-side winglet significantly reduces the local heat transfer near the pressure side and the leakage flow rate in comparison with a flat tip. Jin and Goldstein [7] and Wheeler and Sandberg [8] observed that the gap flow undergoes a laminar-to-turbulent transformation after reattachment. In a facility with high-speed linear cascade, Zhang and He [9] applied transient infrared thermography to investigate the shock wave structure and the corresponding heat transfer over the blade tips. They revealed that the formation of stripes denotes the changes in turbulent viscosity due to the acceleration and deceleration of the flow between the shock waves. Zhang et al. [10] showed that a $9 \%$ decrease in the end-wall boundary layer thickness and inlet turbulence did not affect the tip flow pattern due to flow acceleration near the gap inlet. Zhang et al. [11] and Zhou [12] indicated that the relative motion of the blade and casing provides a force tangential to the tip flow, which causes a reverse vortex inside and attenuates the leakage while maintaining the flow characteristics.

More studies have been conducted using a simplified gap configuration due to the complexity of actual blades and the limitations of experimental conditions. Wheeler and Saleh [13] conducted a numerical and experimental study on transonic flow based on the simplified gap configuration to analyze the effects of the pressure ratio on the shock wave structure and tip flow leakage. The results demonstrated that as the ratio of the inlet total to the exit static pressure increases, the peak Mach number of the tip flow slowly increases, and the normal shock gradually moves downstream. Shyam and Ameri [14] computationally investigated the effects of tip geometry on aerodynamic loss and tip heat transfer. Their results indicated that a divergence pathway could weaken the intensity of the shock and fluctuations of the heat transfer.

The flow separation and reattachment at the gap inlet are the main features of the gap flow that determine the downstream structures. However, there is a paucity of quantitative investigations of flow separation. Moore and Elward [15] studied shock formation in transonic tip flow resulting from flow overexpansion at the inlet of a water tunnel. They developed a formula to predict the location of the reattachment point and angle of the first oblique shock wave. Chen et al. [3] observed that the separation bubble length decreased when the tip flow changed from subsonic to supersonic flow. Furthermore, a numerical study conducted by Wheeler et al. [4] suggested that the acceleration near the pressure side promotes the reattachment and decreases the length and height of the separation bubble by half when the Mach number at the tip gap exit increases from 0.1 to 1 .

The Reynolds number is one of the most important parameters in fluid dynamics. However, the variation of the gap flow fields with respect to the gap configuration and Reynolds number remains unknown. Using oil flow visualizations and static pressure measurements, Key and Arts [16] examined the aerodynamic flow fields of flat and squealer tip gaps under transonic conditions. The results showed that the squealer tip gap was relatively more sensitive to variations in the Reynolds number than the flat gap. Direct numerical simulations (DNS) were applied by Wheeler and Sandberg [8] to investigate the effects of the Reynolds number on the flow fields within a simplified gap configuration with a contraction ratio of 6 and an aspect ratio of 5 . They observed that the inlet separation zone was insensitive to the value of the Reynolds number $\left(1 \times 10^{4}<\operatorname{Re}<2 \times 10^{4}\right)$, and the most apparent effect of the Reynolds number was the change in the separation region resulting from the shock wave boundary layer interactions. Gao et al. [17] computationally investigated the effects of the gap height on the aerodynamic performance of a highperformance turbine blade tip. The results showed that an increase in the tip gap height delays the shock reflection and strengthens its intensity, which in turn reduces the mass flow density (i.e., mass flow rate per unit area).

As mentioned previously, tip gap flows are inevitable in turbomachinery, and transonic gap flow is a common occurrence in modern gas turbines. The inlet separation zone is the primary feature of transonic gap flow that directly determines the flow acceleration, reattachment, shock generation, reflection, and leakage loss characteristics. Although a few studies of transonic tip flows have been conducted, their physical details remain significantly less understood than those of subsonic tip flow. Specifically, the variations in the flow fields resulting from changes in the gap configuration, pressure ratio, and Reynolds number are not clear and, thus, require further research.

The present study is aimed at examining transonic tip flow characteristics and identifying the effects of the Reynolds number on the flow field. Flow measurements were performed on a tip model using particle image velocimetry (PIV) and Schlieren and oil flow visualizations to investigate the flow characteristics and validate a CFD model. The prediction was performed for a simplified tip geometry, wherein the tip gap corresponded to a channel with a constant area. Furthermore, numerical analyses were performed and provided a deeper understanding of the flow mechanism in the tip gap. The rest of the manuscript is organized as follows. The tested model and measurement methods are described in Section 2. The setup of the prediction and grid independence are introduced in Section 3. In Section 4, the experimental results for a typical case and verification of the CFD model are presented, and a discussion of the impacts of the Reynolds number on the separation region, shock waves, 
discharge coefficients, and skin friction is provided. Finally, a few important conclusions are presented in Section 5.

\section{Experimental Setup}

The experiments in this study were performed using a simplified tip geometry that has been applied worldwide by researchers, such as Ameri et al. [18] and Moore et al. [19], to investigate gap flow. Figure 2 shows the side and top views of the typical model tested; the gap height was $10 \mathrm{~mm}$, and the gap width was $30 \mathrm{~mm}$. The actual blade and casing corresponded to a cuboid boss and the upper wall, and a constantarea channel and a sharp inlet corner simulated the tip gap of the turbine blade. However, the pressure side of the tested model had a rounded corner with a radius of approximately $0.3 \mathrm{~mm}$ due to machining and processing. Based on the theory by Rouse [20], the effect of the cuboid boss height on the gap flow fields can be ignored when the contraction ratio exceeds 6. In this experiment, the contraction ratio was greater than 14. The tip flow in the actual blade tip gap was relatively independent because it was mainly affected by the pressure difference and was perpendicular to the local mainstream direction of the blade. Therefore, the flow field structure in the simplified gap could be considered to realistically represent the flow in the midrear part of the blade tip, and the study of its flow mechanism is of significant theoretical and practical importance.

The experiments were conducted in the supersonic wind tunnel of the National University of Defense Technology. The pressure upstream of the wind tunnel equaled atmospheric pressure and remained constant. A spherical vacuum tank was connected downstream of the wind tunnel. The cross-sectional area of the wind tunnel test section was 200 $\times 200 \mathrm{~mm}^{2}$, and the test section had four $400 \times 200 \mathrm{~mm}^{2}$ observation windows. In these experiments, the pressure downstream of the wind tunnel was less than $10 \mathrm{kPa}$. Given that the gap height $(10 \mathrm{~mm})$ was less than the nozzle throat $(45 \mathrm{~mm})$ of the wind tunnel, the nozzle was not started during the experiment. Based on the gap height and exit conditions, the Reynolds number in the typical experiment was

$$
\operatorname{Re}=\frac{\rho V h}{\mu},
$$

where $\rho=0.61 \mathrm{~kg} / \mathrm{m}^{3}, V=361.91 \mathrm{~m} / \mathrm{s}, h=10 \mathrm{~mm}$, and $\mu=$ $1.45 \times 10^{-5} \mathrm{~Pa} \cdot \mathrm{s}$. Therefore, the Reynolds number was approximately $1.5 \times 10^{5}$.

The measurements were performed in the gap between the cuboid boss and the upper wall. During the operation of the wind tunnel, the incoming flow was parallel to the casing and bottom walls of the gap. The velocity data and flow structure in the central section were obtained through PIV and Schlieren photography, respectively. Additionally, oil flow visualizations were performed to reveal the streamline distributions on the bottom wall of the gap; specifically, the inlet separation bubble characteristics were analyzed. The CFD predictions were compared with the experimental results to validate the accuracy of the simulations.
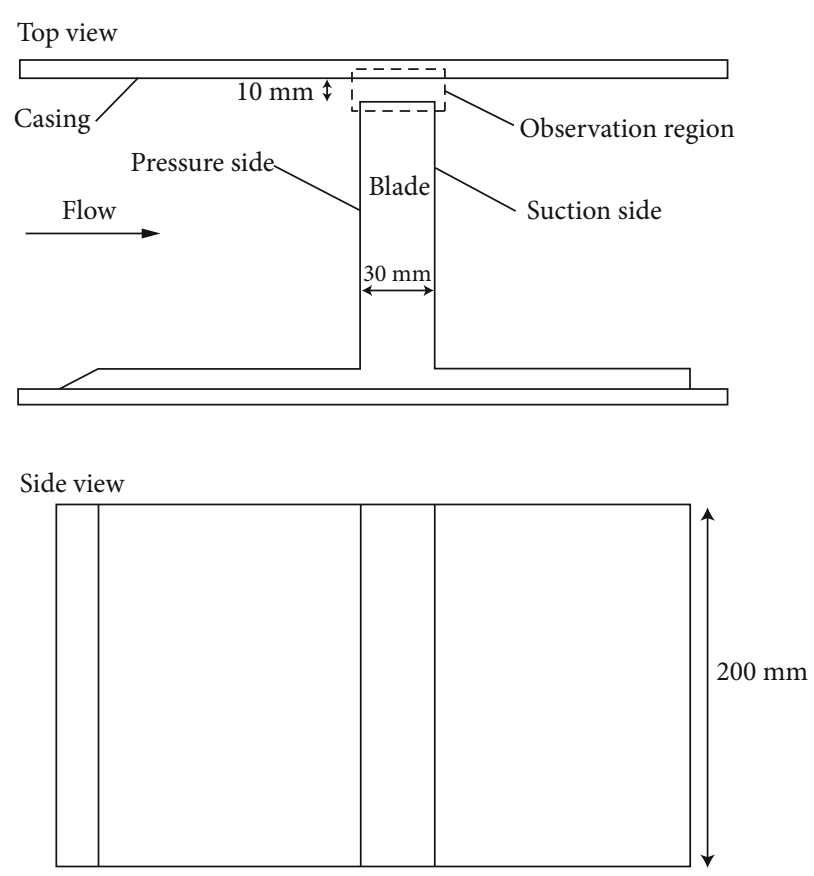

FIGURE 2: Schematic of the experimental Q-3-D gap model.

Titanium dioxide $\left(\mathrm{TiO}_{2}\right)$ was adopted as the tracer particle for the PIV measurements and was uniformly seeded into the wind tunnel via a high-pressure air source. A doublepulsed laser with a wavelength of $532 \mathrm{~nm}$ was employed to illuminate the tracer particles, and an IMPERX chargecoupled device (CCD) camera with a speed of $2 \mathrm{fps}$ captured images of the flow. A synchronizer was employed to control the time series between the CCD camera and the laser. The time delay between the two frames was set to $500 \mathrm{~ns}$. More details regarding the PIV measurement are provided in [21]. The upper part of the cuboid boss was fabricated from glass to minimize laser scattering and was fixed to the base with an ultraviolet-cured adhesive to allow for light passage.

A Z-type configuration Schlieren setup was employed, and the focal length of the concave mirror used in the system was set to $2 \mathrm{~m}$. A high-speed camera with a focal length of $500 \mathrm{~mm}$ and a speed of $1000 \mathrm{fps}$ was used to obtain clearer images of the gap flow fields. Specifically, the oil flow visualizations were captured with the CCD camera and were performed during the operation of the wind tunnel to prevent oil from backflowing due to suspension in the wind tunnel. The experimental results were obtained after the wind tunnel operated smoothly for more than $5 \mathrm{~s}$.

\section{Computational Setup}

3.1. Solver, Grid, and Boundary Conditions. CFD predictions using FLUENT software were performed on a twodimensional model. Given that the gap flow was transonic, a density-based solver was adopted. Furthermore, a secondorder upwind scheme was utilized to solve the steady compressible Reynolds-averaged Navier-Stokes equations. The results predicted by the Spalart-Allmaras, $k$-omega, and $k-$ $\varepsilon$ turbulence models were compared, and the results showed 


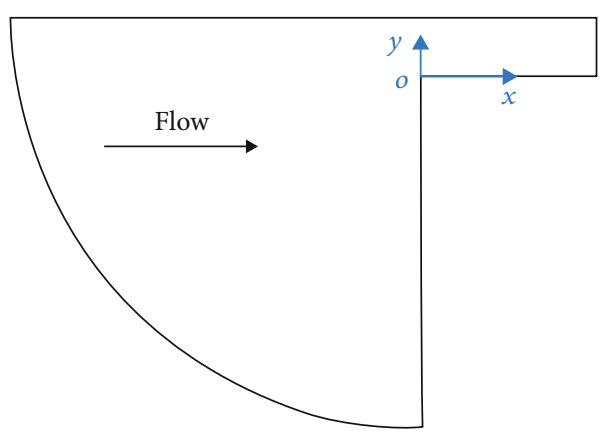

(a)

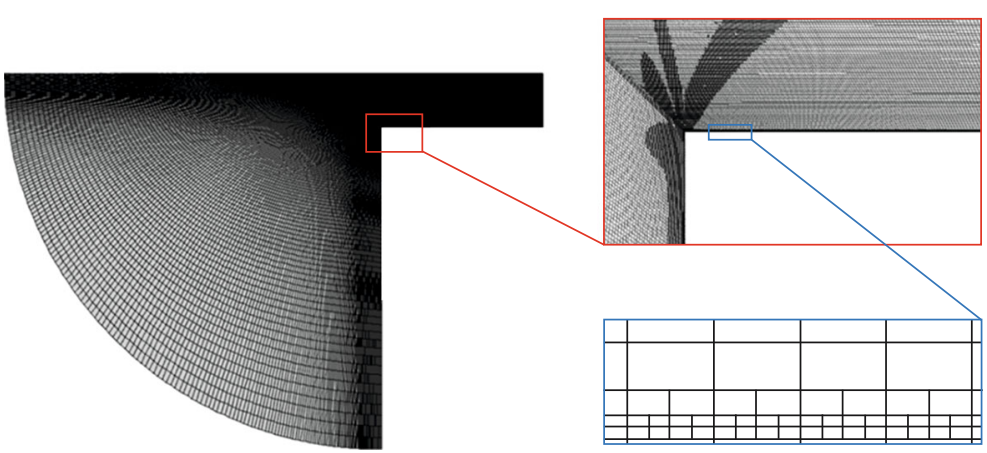

(b)

Figure 3: Computational domain and the typical mesh applied: (a) simplified 2-D computational domain and (b) mesh for the simplified 2-D gap.

that the predictions of the Spalart-Allmaras model agreed well with the measurements in the region near the pressure side of the tip gap.

Figure 3(a) shows that the computational domain was identical to the experimental gap configuration described in Figure 2. In the computational domain, the tip gap and the sharp corner of the inlet corresponded to a constant-height rectangular channel and the pressure-side edge of the blade, respectively. In the CFD verification, the sharp inlet corner was replaced by a rounded corner with a radius of $0.3 \mathrm{~mm}$ to be consistent with the experimental conditions. The numerical studies indicated that when the radius of the inlet corner was less than $0.5 \mathrm{~mm}$, the flow field was not sensitive to the rounded corner. The contraction ratio of the computational domain was seven, which satisfies the criterion (i.e., greater than six) for ignoring its effect on the tip flow fields. Figure 3(a) also provides the Cartesian coordinate system employed in this study, wherein the $x$-direction was along the tip surface, the $y$-direction was perpendicular to the tip surface, and the origin was at the sharp corner.

The mesh was constructed in commercial software, Pointwise, as shown in Figure 3(b). Given that the flow parameters varied significantly in the gap, a solutionadaptive mesh refinement was used in the region having high density gradients. After refinement, the $y+$ values near the wall were less than two. Therefore, no wall function was used.

The numerical experiments suggested that an isothermal or adiabatic wall boundary condition had a negligible effect on the flow structures, such as the shock waves and separation bubble. This study applied a no-slip adiabatic wall condition to all the boundaries. The experimental conditions for the typical simulation case are listed in Table 1, which also shows that, in the other simulation cases, $P_{0}^{*}$ and $h^{*}$ varied between 0.1 and 100. The calculations were considered to converge when the difference in the inlet and outlet mass flow rates was less than $0.1 \%$ for 1000 successive iterations.

3.2. Grid Independence Verification. Models with a total of 50 000, 100 000, 200000 , and 500000 grid nodes were obtained by refining the grid nodes in the gap. Figure 4 provides a comparison of the average friction coefficients $(C \bar{f})$ on the gap surfaces of the four grids. Specifically, the $\mathrm{C} \overline{\mathrm{f}}$ decreased from 0.00125 to 0.00118 (5.6\%) when the number of nodes
TABLE 1: Simulation setup parameters.

\begin{tabular}{lc}
\hline Typical simulation condition & Other simulation conditions \\
\hline$P_{\text {0typ }}=101 \mathrm{kPa}$ & $0.1-100 P_{\text {otyp }}$ \\
$T_{\text {typ }}=300 \mathrm{~K}$ & $T_{\text {otyp }}$ \\
$\mathrm{PR}_{\text {typ }}=100: 1$ & $\mathrm{PR}_{\text {typ }}$ \\
$\mathrm{Tu}_{\text {typ }}=1 \%$ & $\mathrm{Tu}_{\text {typ }}$ \\
$\mathrm{Re}_{\text {typ }}=147920$ & $0.1-100 \mathrm{Re}_{\text {typ }}$ \\
$h_{\text {typ }}=10 \mathrm{~mm}$ & $0.1-100 h_{\text {typ }}$ \\
$w_{\text {typ }}=30 \mathrm{~mm}$ & $0.1-100 w_{\text {typ }}$ \\
\hline
\end{tabular}

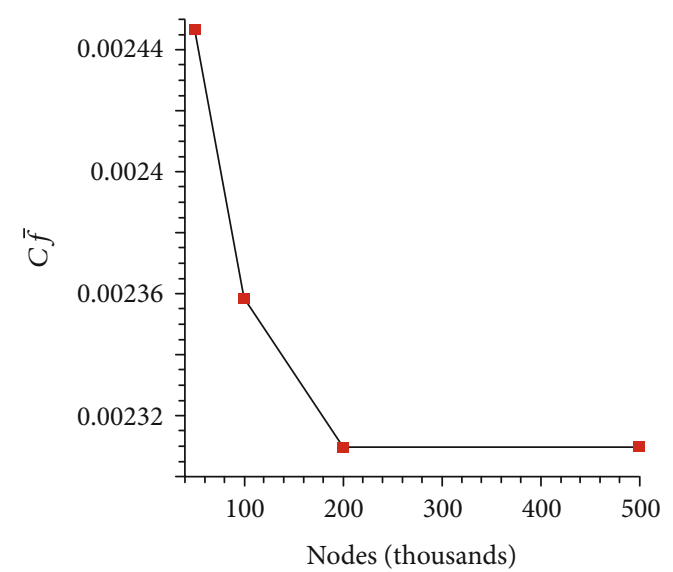

FIGURE 4: Grid dependency analysis.

increased from 50000 to 200000 . When the number of grid nodes exceeded 200000 , the $\mathrm{C} \bar{f}$ of the tip surface remained unchanged, thus satisfying the requirements for grid independence. On this basis, a 200000 mesh was chosen for the CFD prediction in this study to provide a balance between computational accuracy and cost.

\section{Results and Discussion}

4.1. Experimental Results. The experimental and numerical results of the gap flow field for a gap height of $10 \mathrm{~mm}$ and an aspect ratio of 3 are depicted in Figure 5. Specifically, the 


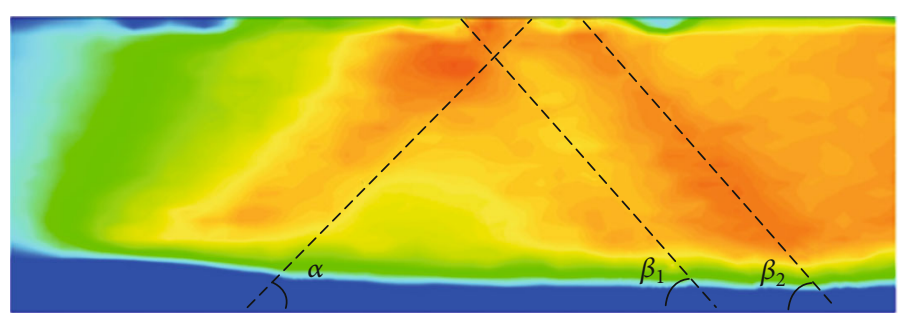

(a)

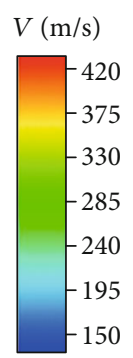

50

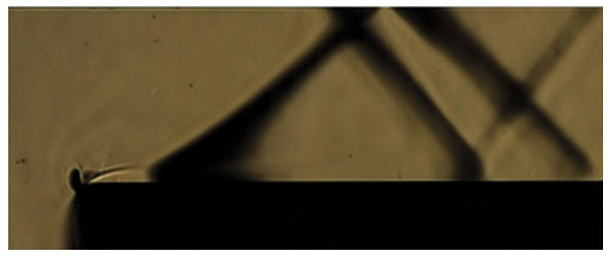

(b)

Figure 5: (a) Velocity contours and (b) Schlieren image obtained experimentally ( $w=30 \mathrm{~mm}, h=10 \mathrm{~mm})$.

PIV measurement in Figure 5(a) shows the flow structure and velocity contour in the gap. As the flow entered the tip gap, it separated at the inlet corner and decreased the flow area of the passage; therefore, the subsonic flow accelerated rapidly to reach sonic speed near the top of the separation bubble. Subsequently, the expanded channel formed by the separation bubble and casing wall surface forced the supersonic airflow to accelerate continuously. Compression waves formed owing to the change in the curvature of the boundary layer near the reattachment point, which, in turn, coalesced into an oblique shock wave. The shock wave and boundary layer interactions on the casing wall led to a boundary layer separation, thus forming two shock waves that reflected between the casing and tip and, finally, exited the gap.

The Schlieren image shown in Figure 5(b) illustrates the flow pattern of the inlet separation and shock reflections previously described. Notably, a darker pincer region appeared at the front of the separation bubble, resulting from an optical deflection caused by the significant change in the density gradient and the abrupt acceleration and turning of the airflow at the entrance. The outer edge of the separation bubble was clear, and its thickness was minimal, indicating that the shear layer was relatively stable and developed slowly; this occurred because the gap size and Reynolds number were relatively small, while the favorable pressure gradient was large. Nevertheless, the shear layer widened where the shock wave was generated. Based on the DNS results of Wheeler and Sandberg [8], the vortex structures began to break up where the shock wave formed, and the compression waves were complicated and accompanied by oscillations.

Figure 6 shows the stripes of the oil flow on the bottom wall of the gap and the streamlines near the wall. These stripes are normal to the flow direction and result from flow separations and reattachments. The separated airflow stagnated at the centerline L2 of the reattachment region, and streamlines developed on both sides in the forward and backward directions, resulting in a broad white stripe. The secondary separation occurred at L1, and the streamlines on both sides converged to the middle to form a noticeable bulge. The origins of L3 and L4 were the separations caused by the shock wave boundary layer interactions. However, they were significantly smaller than the separation bubble at the entrance.

The measurements from all the experiments corresponded well. All the flow reattachments occurred at approximately one-third of the gap width. In the PIV image, the

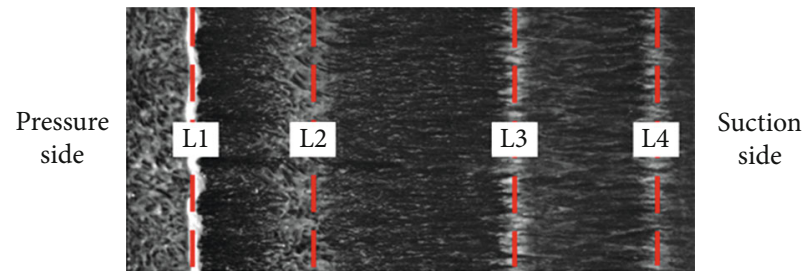

(a)

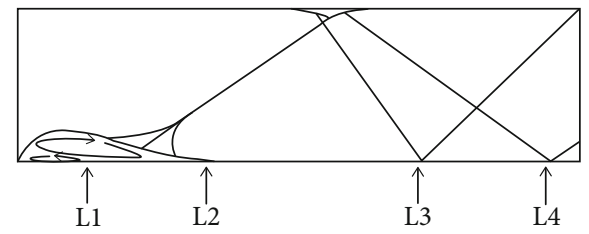

(b)
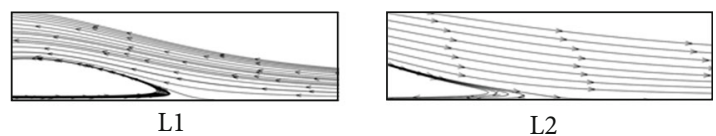

L2

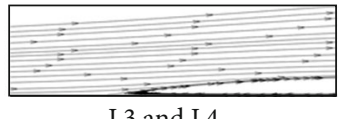

L3 and L4

(c)

Figure 6: Oil flow stripes and schematic diagrams: (a) results of the oil flow visualization on the bottom wall; (b) schematic of the flow field; (c) schematics of the streamlines near the wall.

angle $\alpha$ of the first shock wave was approximately $51^{\circ}$. The angles $\beta_{1}$ and $\beta_{2}$ of the two reflecting shock waves were approximately $56^{\circ}$ and $54^{\circ}$, respectively. These angles were essentially the same as those obtained by the Schlieren imaging. Furthermore, the positions of the shock wave reflections between the two images corresponded extremely well. These comparisons between the experimental measurements demonstrated the validity of the results.

Figure 7 shows the oil flow results for gap configurations having different widths and heights. The images on the left show the original experimental results, and the images on the right are normalized by the gap heights of those on the left. In the three test conditions shown in Figure 7(a), the gap width was $30 \mathrm{~mm}$, and the gap height increased from $5.8 \mathrm{~mm}$ to $12.2 \mathrm{~mm}$. As the gap height increased, the shock wave reflections were delayed, and the secondary flows and the sizes of the separation bubbles increased. After 


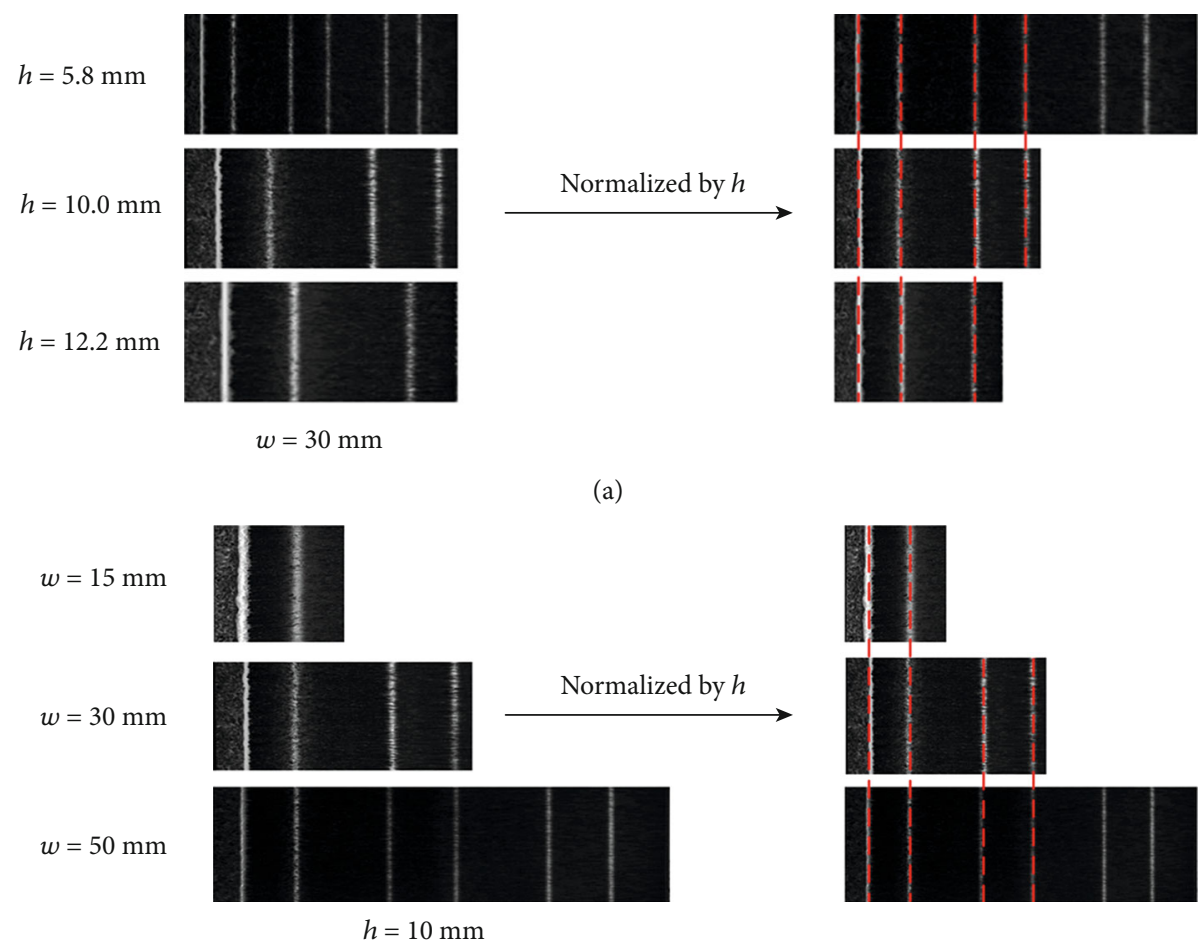

(b)

FIGURE 7: Results of the oil flow visualization for different gap (a) heights and (b) widths.

TABLE 2: Length of the secondary flows and separation bubbles for different gap widths and heights.

\begin{tabular}{lccccc}
\hline Gap width $(\mathrm{mm})$ & Gap height $(\mathrm{mm})$ & Secondary flow $(\mathrm{mm})$ & $\begin{array}{c}\text { Normalized } \\
\text { secondary flow }\end{array}$ & $\begin{array}{c}\text { Separation bubble } \\
\text { length (mm) }\end{array}$ & $\begin{array}{c}\text { Normalized separation } \\
\text { bubble length }\end{array}$ \\
\hline 30 & 5.8 & 1.99 & 0.34 & 5.47 & 0.94 \\
30 & 10 & 3.58 & 0.36 & 9.45 & 0.95 \\
30 & 12.2 & 4.38 & 0.36 & 11.70 & 0.96 \\
50 & 10 & 3.51 & 0.35 & 9.38 & 0.94 \\
30 & 10 & 3.58 & 0.36 & 9.45 & 0.95 \\
15 & 10 & 3.68 & 0.37 & 9.64 & 0.96 \\
\hline
\end{tabular}

TABle 3: Comparisons of the separation bubble lengths obtained from the experiments and simulations.

\begin{tabular}{lcccccccc}
\hline $\begin{array}{l}\text { Gap width } \\
(\mathrm{mm})\end{array}$ & Gap height $(\mathrm{mm})$ & $\begin{array}{c}\text { Experimental } \\
\text { length }(\mathrm{mm})\end{array}$ & $k-\varepsilon(\mathrm{mm})$ & Deviation $(\%)$ & $k$-omega $(\mathrm{mm})$ & Deviation $(\%)$ & S-A (mm) & Deviation (\%) \\
\hline 30 & 10 & 9.45 & 8.00 & 15.34 & 9.71 & 2.75 & 9.58 & 1.41 \\
30 & 7 & 6.74 & 5.26 & 20.10 & 6.45 & 4.90 & 6.64 \\
50 & 10 & 9.38 & 8.02 & 15.00 & 9.72 & 2.86 & 9.57 & 2.03 \\
\hline
\end{tabular}

normalization, the separation bubble and the secondary flow lengths for the different-height gaps became the same, 0.95 and 0.35 , respectively. Further details are provided in Table 2. The positions of the shock wave boundary layer interactions also corresponded well. These results indicate that flow similarity exists between flow fields with different gap heights at experimental Reynolds numbers. In the leftside images in Figure $7(\mathrm{~b})$, the gap height remained at $10 \mathrm{~mm}$, and the width varied from $15 \mathrm{~mm}$ to $50 \mathrm{~mm}$. The increased gap width resulted in an increasing number of shock wave reflections, but it did not affect the flow similarity.

4.2. CFD Verification. The accuracy of the FLUENT model adopted in this paper was validated based on the experimental measurements. Table 3 shows a comparison of the separation bubble lengths predicted by the three turbulence models and the experimental measurements. Concerning the different gap configurations (aspect ratios of $5: 1,3: 1$, and $30: 7$ ), the $k-\varepsilon$ model underestimated the separation bubble length 


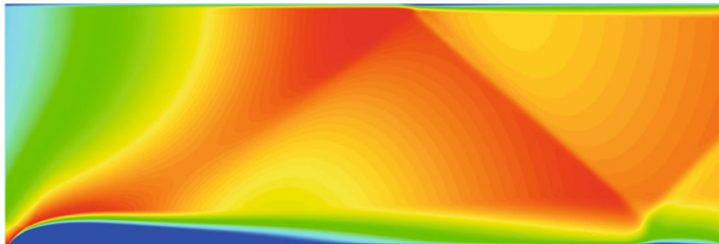

(a)

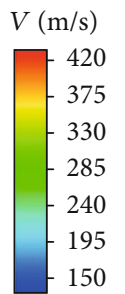

150

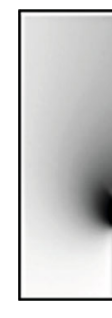

Figure 8: (a) Velocity contour and (b) Schlieren image obtained from the simulation $(w=30 \mathrm{~mm}, h=10 \mathrm{~mm})$.

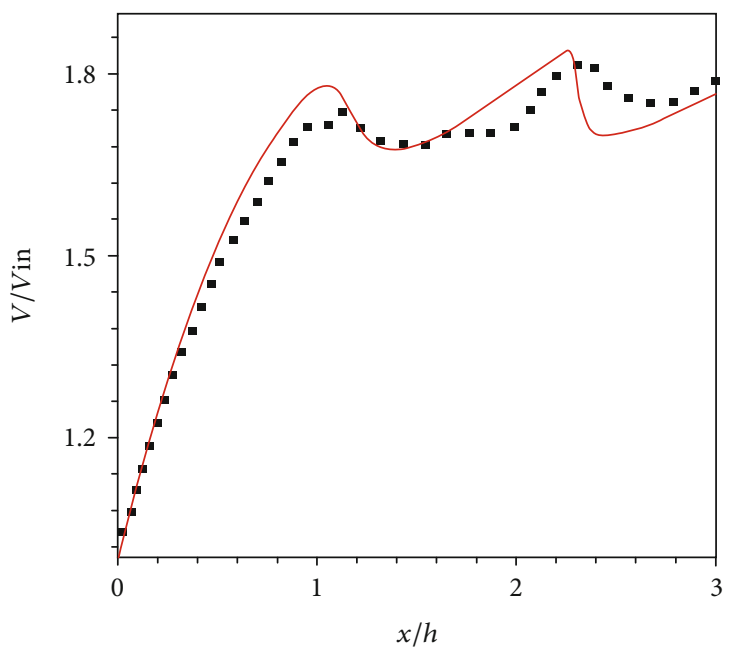

- Experiment

- CFD

Figure 9: Dimensionless velocity distributions at the centerline obtained from the CFD model and experiment.

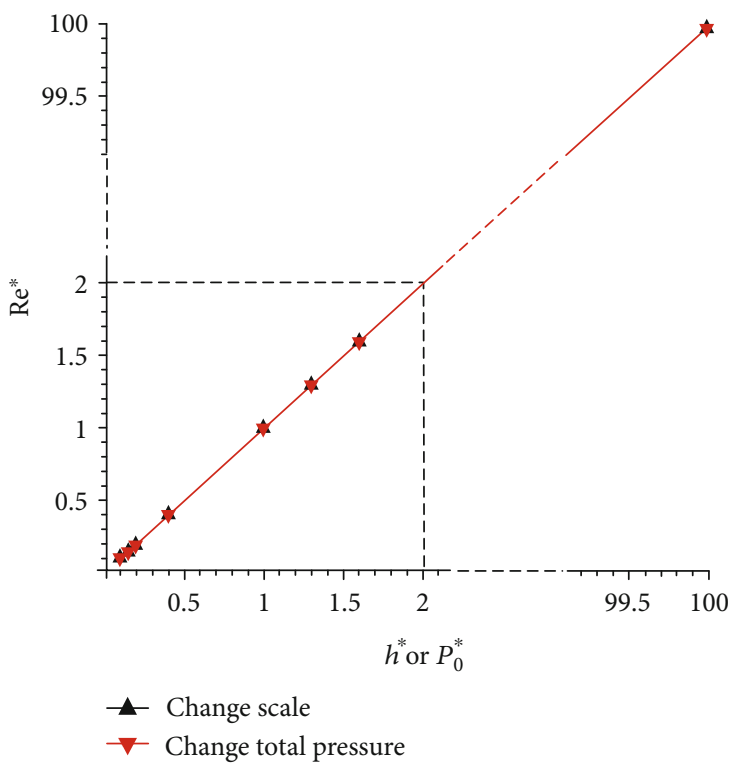

Figure 10: Variation in the Reynolds numbers with respect to gap size and total pressure.

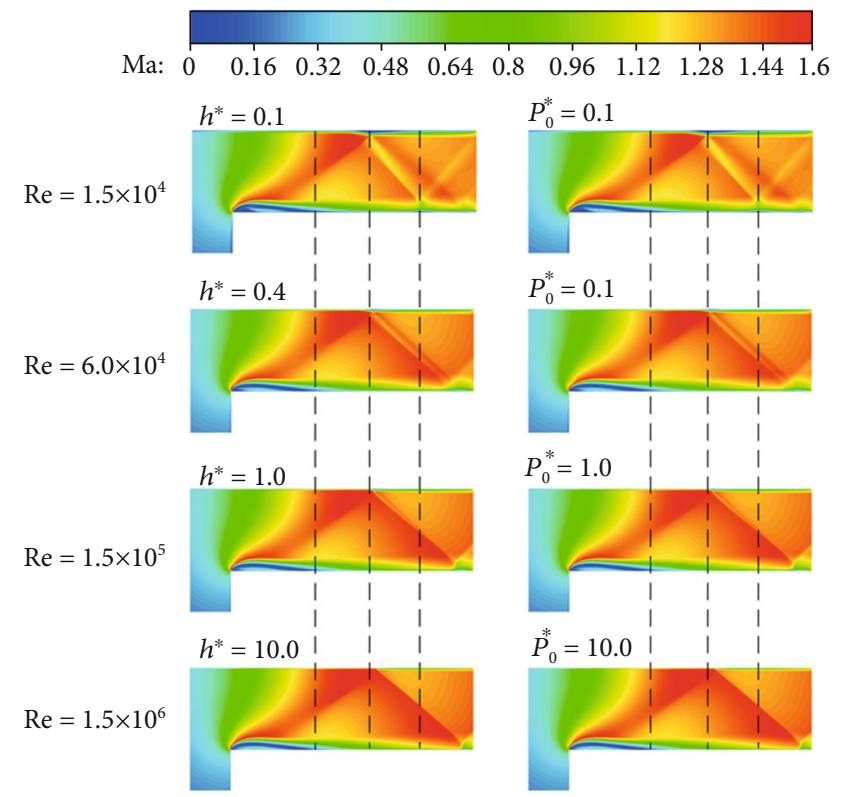

Figure 11: Mach number contours for different gap sizes and total pressure values.

by approximately $20 \%$ and failed to predict the secondary separation near the gap inlet. Both the $k$-omega and the Spalart-Allmaras turbulence model agreed reasonably well with the experimental results, and the Spalart-Allmaras model more accurately predicted the separation bubble length, providing deviations of less than $2.5 \%$. Therefore, the Spalart-Allmaras turbulence model, which has been widely used and proven to be effective in the prediction of tip flow, especially for the separation region near the pressure-side edge [22], was selected for application in this study.

The CFD predictions for the velocity and $x$-density gradient for the typical tested condition are shown in Figure 8. The simulation provided typical flow characteristics, such as inlet separation region, flow acceleration, and shock reflections, which were substantially similar to the measurements. Figure 9 compares the computed and measured normalized velocity distributions at the central line of the typical condition. As the flow crossed the tip gap, the velocity near the gap inlet increased rapidly and then underwent multiple decelerations and accelerations. The three peak velocity values measured corresponded to three oblique shocks, which are also shown in Figure 5. The converged shock wave and the second reflected shock wave were obvious, while the 


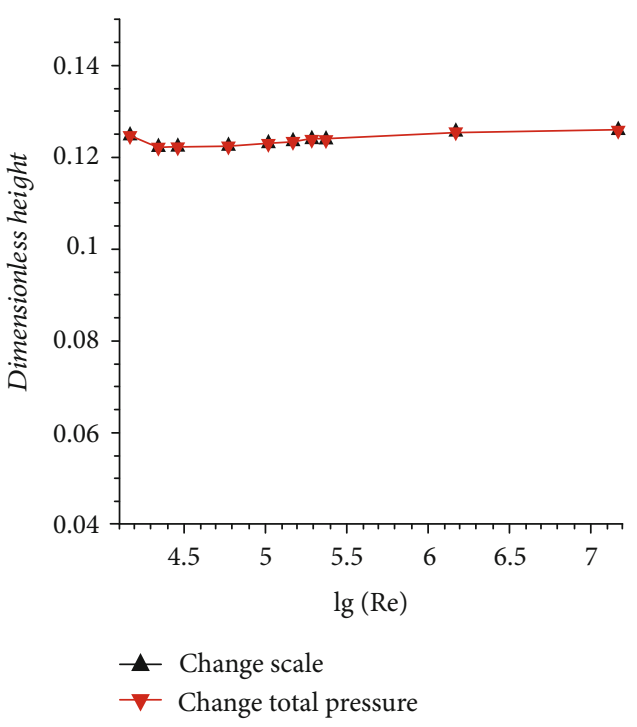

(a)

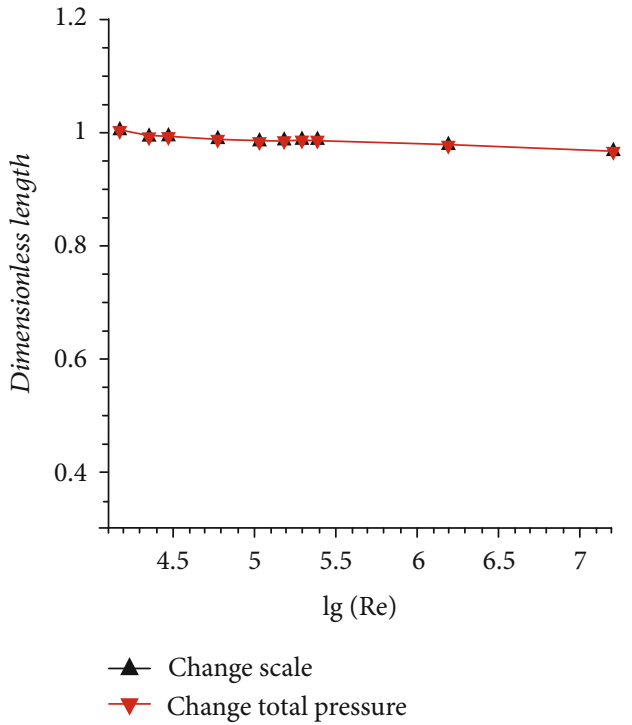

(b)

FIGURE 12: Comparison of the dimensionless separation bubble (a) heights and (b) lengths obtained by changing the scale and total pressure.

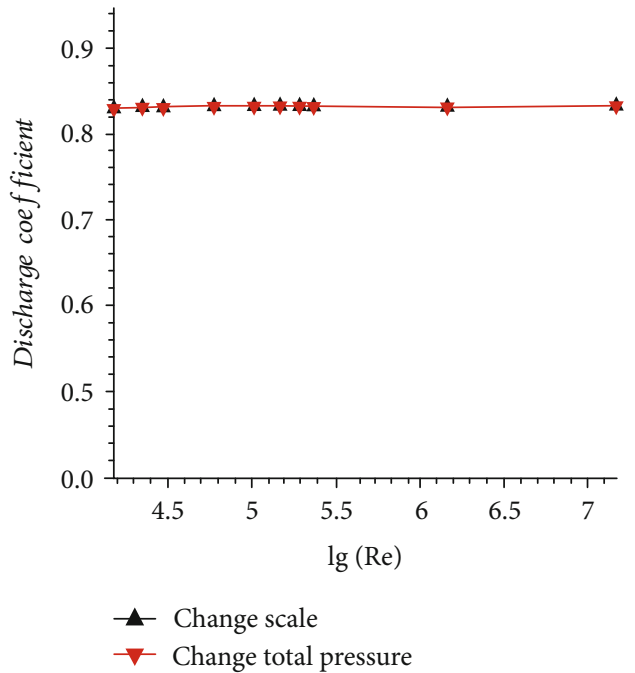

FIGURE 13: Comparison of the discharge coefficients obtained by changing the scale and total pressure.

first reflected shock was weaker. The experimental data agreed extremely well with the CFD predictions for the separation region, converged shock wave, and second reflected shock wave. Two reflected shock waves occurred experimentally; however, only one appeared in the CFD results, resulting from the CFD underestimating the separation bubble caused by the shock wave-boundary layer interaction. However, this study was primarily focused on the inlet separation region and shock wave at the reattachment region, and the accuracy of the CFD predictions satisfied the requirements in those regions.

4.3. Separation Bubble and Discharge Coefficient at Different Reynolds Numbers. Based on the gap height and exit conditions, the Reynolds numbers for the CFD simulations were in the range of $1.5 \times 10^{4}$ to $1.5 \times 10^{7}$. Figure 10 shows that, relative to the typical case $\left(P_{\text {typ }}=101 \mathrm{kPa}, T_{0}=300 \mathrm{~K}, \mathrm{PR}=\right.$ $\left.100: 1, h_{\text {typ }}=10 \mathrm{~mm}\right)$, the Reynolds number varied when the total pressure or the gap height changed. Figure 11 shows the Mach number distributions when $P_{0}^{*}$ and $h^{*}$ were $0.1,0.4$, 1 , and 10. For the convenience of comparison, the gap size values were normalized by the gap height.

Figure 11 shows that when the Reynolds number was maintained constant, and the gap size or total pressure changed; the tip flow characteristics were the same. Furthermore, variations in the Reynolds number did not noticeably alter the flow pattern; however, the local Mach number and shock waves varied significantly. At a low Reynolds number, the separation on the casing wall was evident after the shock wave boundary layer interactions due to the relatively weak momentum in the boundary layer. This, in turn, resulted in the formation of two reflecting shock waves. As the Reynolds number increased, the tip flow exhibited an enhanced ability to resist the adverse pressure gradients, and thus, the two reflecting shock waves merged into one. The shock wave then moved backward, and its intensity increased.

In this study, the flow in the gap was choked. The mass flow rate is determined by the area of the throat and the total temperature and total pressure at the gap entrance. The discharge coefficient is defined as follows:

$$
C_{V}=\frac{\dot{m}}{h l_{z}\left(P_{0} / \sqrt{T_{0}}\right) \sqrt{\gamma / R}((\gamma+1) / 2)^{-(\gamma+1) /(2(\gamma-1))}},
$$

where $\dot{m}$ denotes the actual tip flow rate in the gap, $h$ denotes the gap height, $l_{z}$ denotes the spanwise length and is $1 \mathrm{~m}$ by default, and $P_{0}$ and $T_{0}$ denote the total pressure and total temperature at the entrance of the gap, respectively. 


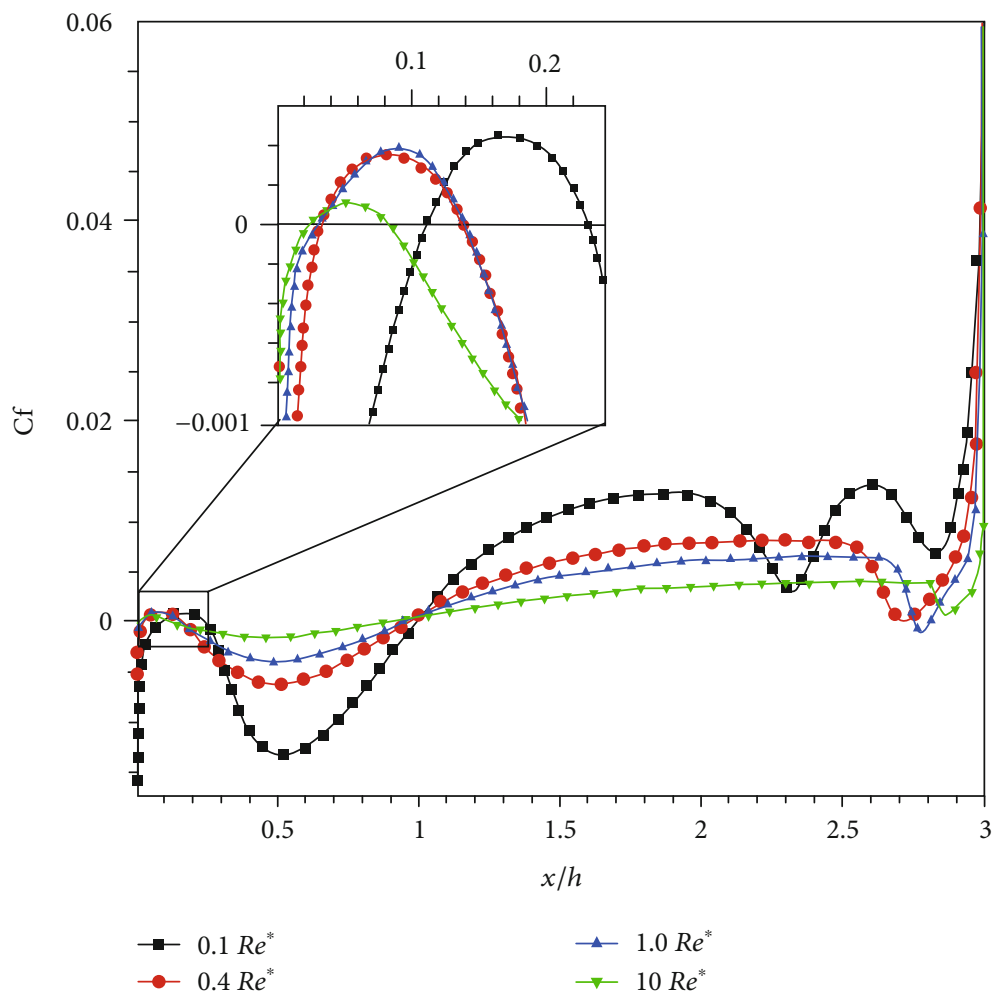

FIgURE 14: Effect of the Reynolds number on the friction coefficient of the bottom wall.

Furthermore, $R$ denotes the gas constant $(287 \mathrm{~J} /(\mathrm{kg} \cdot \mathrm{K}))$, and $\gamma$ denotes the specific heat ratio (1.4).

Figure 12 shows the effects of the Reynolds number on the separation length and height. At a constant Reynolds number, the difference between the separation bubble size obtained by changing the gap scale or total pressure was minuscule. The results shown in Figures 11 and 12 demonstrate that the flow similarity existed as long as the Reynolds number remained constant, which illustrates that the Reynolds number is the key parameter, not gap size or pressure, that determined the tip flow fields. The height and length of the dimensionless separation bubbles were approximately 0.125 and 0.95 and did not noticeably change with variations in the Reynolds number; the deviations between the maximum and minimum values were $4.1 \%$ and $5.3 \%$, respectively. This indicates that the shape of the separation bubble was not sensitive to changes in the Reynolds number within the range of $1.5 \times 10^{4}$ to $1.5 \times 10^{7}$. The variation in the discharge coefficient with respect to the Reynolds number is shown in Figure 13. As the Reynolds number increased, the discharge coefficient remained constant at 0.83 because the dimensionless height of the separation bubble was nearly unchanged.

4.4. Skin Friction Coefficient at Different Reynolds Numbers. The skin friction coefficient is determined by the shear stress on the bottom wall of the gap and is defined as follows:

$$
\mathrm{Cf}=\frac{\tau_{w}}{(1 / 2) \rho_{\text {in }} V_{\text {in }}^{2}},
$$

where $\tau_{w}$ denotes the wall shear stress and $\rho_{\text {in }}$ and $V_{\text {in }}$ are determined by the density and velocity at the gap inlet, according to

$$
\begin{gathered}
\rho_{\text {in }}=\frac{\int_{0}^{h} \rho d y}{h}, \\
V_{\text {in }}=\frac{\int_{0}^{h} V d y}{h},
\end{gathered}
$$

Figure 14 shows the distributions of the skin friction coefficients on the tip wall. There was a strong backflow in the inlet separation bubble. Hence, Cf was negative in most of the region, but notably, the Cf near the inlet $(0 \leq x / h \leq 0.23)$ was positive. This resulted from the secondary vortex, which was opposite the direction of the primary recirculation, as shown in Figure 6. The flow stagnated at the reattachment point, and the Cf corresponded to 0 . The flow in the boundary layer gradually accelerated downstream of the reattachment point, and the Cf was positive and increased slowly. The flow near the wall decelerated, stagnated, and even reversed due to the shock wave boundary layer interactions, leading to the fluctuation in the Cf. As the Reynolds number varied from $0.1 \mathrm{Re}^{*}$ to $10 \mathrm{Re}^{*}$, the magnitude of the $\mathrm{Cf}$ decreased gradually, and the effect of the shock wave boundary layer interactions on the Cf decreased.

Figure 15 shows the variations in the average friction coefficient. The average skin friction coefficient of the gap decreased as the Reynolds number increased. Additionally, a linear relationship was demonstrated between the logarithm of the average skin friction coefficient and the logarithm of the Reynolds number. Based on the least squares 


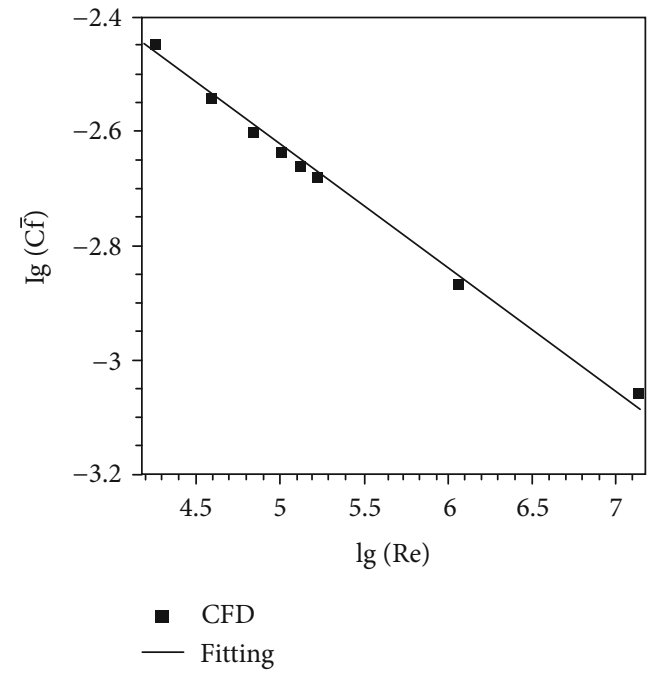

Figure 15: Comparison of the predicted and fitted average friction coefficient.

method, the relationship is as follows:

$$
\lg (\mathrm{C} \overline{\mathrm{f}})=-0.2302 \lg (\mathrm{Re})-1.4336
$$

The maximum deviation between Equation (5) and the simulation was less than 1.4\%, indicating that Equation (5) could be used to estimate the shear stress for blade gap design.

\section{Conclusions}

This study examined the characteristics of transonic tip flow. Specifically, PIV and Schlieren oil flow visualizations were used to obtain the flow field structure for a simplified tip geometry. Additionally, the Spalart-Allmaras turbulence model was applied through FLUENT 18.0 to investigate the aerodynamic characteristics of the tip flows at different Reynolds numbers. The significant conclusions of the study are as follows:

(1) A darker pincer region appeared at the sharp corner due to the rapid acceleration and abrupt turning of the airflow at the entrance. As the flow entered the gap, the boundary layer separated on the pressure side and formed a separation bubble, inside which a small secondary separation vortex occurred. Of the two reflected shock waves, the first was weaker than the second. The size of the separation region increased with the gap height. Significant differences existed between the flow fields for the different gap heights. However, after normalization, the flow fields were similar. Increasing the gap width had a negligible effect on the flow similarity

(2) The Reynolds number, as opposed to the gap size or pressure, is the key parameter determining the tip flow field. For gaps with the same aspect ratio, flow similarity exists as long as the Reynolds number remains constant. This principle could be used to study the flow structures of actual engines. The dimensionless separation bubble size and discharge coefficient were insensitive to the Reynolds number when it was between $1.5 \times 10^{4}$ and $1.5 \times 10^{7}$. The separation bubble length and height were approximately 0.95 and 0.125 times the gap height, respectively. As the Reynolds number increased, the reflected shock waves were delayed, and the shock wave intensity increased

(3) Increasing the Reynolds number reduced the skin friction coefficient on the bottom wall of the gap and the impact of the shock waves on the skin friction coefficient. The skin friction coefficient near the inlet was positive because of the secondary separation within the separation bubble. Furthermore, an excellent linear relationship was demonstrated between the logarithm of the average skin friction coefficient and the logarithm of the Reynolds number. This relationship could be used to estimate the shear stress during blade gap design

\section{Nomenclature}

$P_{0}$ : Total inlet pressure $(\mathrm{kPa})$

$T_{0}: \quad$ Total temperature $(\mathrm{K})$

Tu: Turbulence intensity (\%)

$h$ : $\quad$ Tip gap height $(\mathrm{mm})$

$w$ : Tip gap width $(\mathrm{mm})$

$x, y:$ Distance in the coordinate direction $[\mathrm{mm}]$

$V: \quad$ Velocity $(\mathrm{m} / \mathrm{s})$

$\rho: \quad$ Density $\left(\mathrm{kg} / \mathrm{m}^{3}\right)$

$\gamma: \quad$ Specific heat ratio $(-)$

Ma: Mach number (-)

PR: Inlet total to exit static pressure ratio (-)

Re: Reynolds number (-) $\operatorname{Re}=\rho V h / \mu$

$P_{0}^{*}$ : Relative total pressure (-) $P_{0}^{*}=P_{0} / P_{\text {typ }}$

$h_{0}^{*}$ : Relative gap height $(-) h_{0}^{*}=h / h_{\text {typ }}$

$\mathrm{Re}^{*}$ : Relative Reynolds number (-) $\operatorname{Re}^{*}=\operatorname{Re} / \operatorname{Re}_{\text {typ }}$

Cf: Skin friction coefficient (-) $\mathrm{Cf}=\tau_{w} /(1 / 2) \rho_{\text {in }} V_{\text {in }}^{2}$

$\mathrm{C} \overline{\mathrm{f}}: \quad$ Average skin friction coefficient $\left(\mathrm{m}^{-1}\right) \mathrm{C} \overline{\mathrm{f}}=\int \mathrm{Cf} d x / w$

$C_{V}:$ Discharge coefficient (-) $C_{V}=\dot{m} / h\left(P_{0} / \sqrt{T_{0}}\right) \sqrt{\gamma / R}$ $((\gamma+1) / 2)^{-(\gamma+1) /(2(\gamma-1))}$

\section{Subscript}

typ: Typical

in: Gap inlet

0: Total

\section{Data Availability}

The data used to support the findings of this study are included within the article. 


\section{Conflicts of Interest}

The authors declare there is no conflict of interest regarding the publication of this paper.

\section{Acknowledgments}

This work was supported by the National Natural Science Foundation of China (Grant No. 11802336).

\section{References}

[1] J. D. Denton, “The 1993 IGTI scholar lecture: loss mechanisms in turbomachines," Journal of Turbomachinery, vol. 115, no. 4, pp. 621-656, 1993.

[2] B. Sunden and G. Xie, "Gas turbine blade tip heat transfer and cooling: a literature survey," Heat Transfer Engineering, vol. 31, no. 7, pp. 527-554, 2010.

[3] G. Chen, W. N. Dawes, and H. P. Hodson, "A numerical and experimental investigation of turbine tip gap flow," AIAA Paper, vol. 1993-2253, 1993.

[4] A. P. S. Wheeler, N. R. Atkins, and L. He, "Turbine blade tip heat transfer in low speed and high speed flows," in Volume 3: Heat Transfer, Parts A and B, vol. 133, 2011no. 4.

[5] J. D. Coull, N. R. Atkins, and H. P. Hodson, "Winglets for improved aerothermal performance of high pressure turbines," Journal of Turbomachinery, vol. 136, no. 9, 2014.

[6] A. K. Saha, S. Acharya, R. Bunker, and C. Prakash, "Blade tip leakage flow and heat transfer with pressure-side winglet," International Journal of Rotating Machinery, vol. 2006, 15 pages, 2006.

[7] P. Jin and R. J. Goldstein, "Local mass and heat transfer on a turbine blade tip," International Journal of Rotating Machinery, vol. 9, no. 2, 2003.

[8] A. P. S. Wheeler and R. D. Sandberg, "Numerical investigation of the flow over a model transonic turbine blade tip," Journal of Fluid Mechanics, vol. 803, pp. 119-143, 2016.

[9] Q. Zhang and L. He, "Overtip choking and its implications on turbine blade-tip aerodynamic performance," Journal of Propulsion and Power, vol. 27, no. 5, pp. 1008-1014, 2011.

[10] Q. Zhang, L. He, and A. Rawlinson, "Effects of inlet turbulence and end-wall boundary layer on aerothermal performance of a transonic turbine blade tip," Journal of Engineering for Gas Turbines and Power, vol. 136, no. 5, 2014.

[11] Q. Zhang, D. O. O’Dowd, L. He, M. L. G. Oldfield, and P. M. Ligrani, "Transonic turbine blade tip aerothermal performance with different tip gaps-part I: tip heat transfer," Journal of Turbomachinery, vol. 133, no. 4, 2011.

[12] C. Zhou, "Aerothermal performance of different tips in transonic turbine cascade with end-wall Motion," Journal of Propulsion and Power, vol. 30, no. 5, pp. 1316-1327, 2014.

[13] A. P. S. Wheeler and Z. Saleh, "Effect of cooling injection on transonic tip flows," Journal of Propulsion and Power, vol. 29, no. 6, pp. 1374-1381, 2013.

[14] V. Shyam and A. Ameri, "Comparison of various supersonic turbine tip designs to minimize aerodynamic loss and tip heating," in Volume 7: Turbomachinery, Parts A, B, and C, 2011.

[15] J. Moore and K. M. Elward, "Shock formation in overexpanded tip leakage flow," Journal of Turbomachinery, vol. 115, no. 3, pp. 392-399, 1993.
[16] N. L. Key and T. Arts, "Comparison of turbine tip leakage flow for flat tip and squealer tip geometries at high-speed conditions," Journal of Turbomachinery, vol. 128, no. 2, pp. 213220, 2006.

[17] J. Gao, Q. Zheng, X. Niu, and G. Yue, “Aerothermal characteristics of a transonic tip flow in a turbine cascade with tip clearance variations," Applied Thermal Engineering, vol. 107, pp. 271-283, 2016.

[18] A. A. Ameri, E. Steinthorsson, and D. L. Rigby, "Effect of squealer tip on rotor heat transfer and efficiency," Journal of Turbomachinery, vol. 120, no. 4, pp. 753-759, 1998.

[19] J. Moore, J. G. Moore, G. S. Henry, and U. Chaudhry, "Flow and heat transfer in turbine tip gaps," Journal of Turbomachinery, vol. 111, no. 3, pp. 301-309, 1989.

[20] H. Rouse, Elementary Mechanics of Fluids, Dover Publications, Incorporated, United States, 1946.

[21] Q.-c. Wang, Z.-g. Wang, and Y.-x. Zhao, “An experimental investigation of the supersonic turbulent boundary layer subjected to concave curvature," Physics of Fluids, vol. 28, no. 9, article 096104, 2016.

[22] D. O. O’Dowd, Q. Zhang, L. He, B. C. Y. Cheong, and I. Tibbott, "Aerothermal performance of a cooled winglet at engine representative Mach and Reynolds numbers," Journal of Turbomachinery, vol. 135, no. 1. 